\title{
Anomalous diffusion for overdamped particles driven by cross-correlated white noise sources
}

\author{
S. I. Denisov ${ }^{1,2}$ A. N. Vitrenko, ${ }^{2}$ W. Horsthemke, ${ }^{3}$ and P. Hänggi ${ }^{1}$ \\ ${ }^{1}$ Institut für Physik, Universität Augsburg, \\ Universitätsstraße 1, D-86135 Augsburg, Germany \\ ${ }^{2}$ Sumy State University, 2 Rimsky-Korsakov Street, 40007 Sumy, Ukraine \\ ${ }^{3}$ Department of Chemistry, Southern Methodist University, Dallas, Texas 75275-0314, USA
}

\begin{abstract}
We study the statistical properties of overdamped particles driven by two cross-correlated multiplicative Gaussian white noises in a time-dependent environment. Using the Langevin and FokkerPlanck approaches, we derive the exact probability distribution function for the particle positions, calculate its moments and find their corresponding long-time, asymptotic behaviors. The generally anomalous diffusive regimes of the particles are classified, and their dependence on the friction coefficient and the characteristics of the noises is analyzed in detail. The asymptotic predictions are confirmed by exact solutions for two examples.
\end{abstract}

PACS numbers: 05.40.-a, 05.10.Gg, 02.50.-r 


\section{INTRODUCTION}

Diffusive behavior is intrinsic to many physical, chemical, biological, economical and other systems [1, 2, 3]. One of the most rigorous approaches for its description that takes into account the dynamical origin of diffusive motion is based on the Langevin equation, i.e., the stochastic equation of motion. For a large variety of systems that are stochastically equivalent to free Brownian particles, this equation has the simplest form, namely a constant friction coefficient and additive Gaussian white noise. Such systems exhibit normal diffusion, i.e., the long-time asymptotics of the dispersion of the state parameter are proportional to time.

However, plenty of systems exhibit anomalous diffusion (for a review, see Refs. [4, [5, 6, 4]). In general, there exist several approaches to describe this phenomenon. A first one is based on fractional calculus and involves fractional diffusion equations [8, 9, 10], fractional FokkerPlanck equations [11, 12, 13, 14] or fractional Langevin equations [15, 16, 17]. Yet another other relies on generalized ordinary Langevin equations. The latter is very attractive for the treatment of diffusive behavior, and it is especially informative if those equations can be solved exactly [18, 19]. This approach has been employed to study anomalous diffusion for a variety of systems, including systems described by the generalized Langevin equation with a friction memory kernel [20, 21, 22, 23, 24, 25, 26], free undamped [27, 28, 29] and damped particles [30] driven by additive noise, overdamped particles driven by one [31] and two 32] multiplicative colored noises, and overdamped particles with time-dependent drift driven by additive Gaussian white noise [33].

The Langevin approach is also an efficient tool to study various effects in systems driven by correlated noises. The correlation can exist, for example, if the two noises possess a common origin [34, 35], as in the case of bistable systems where fluctuations of some model parameters are equivalent to the action of correlated additive and multiplicative noises [35].

Such noises play an important role in the description of stochastic resonance [36, 37, 38], nonequilibrium phase transitions [39, 40], and noise-induced transport [41, 42]. We expect that correlated noise will impact significantly the anomalous diffusive behavior. To investigate this problem in the general case of a time-dependent environment, we consider the long-time 
behavior of an overdamped particle governed by the Langevin equation

$$
\lambda(t) \dot{x}(t)=\sum_{i=1}^{2} g_{i}(x(t)) \xi_{i}(t) \quad\left[x(0)=x_{0}\right],
$$

where $x(t)$ is the particle position, $\lambda(t)$ denotes an arbitrary, positive-valued time-dependent friction coefficient, and $g_{i}(x)$ are deterministic functions that characterize the statedependent action of Gaussian noises $\xi_{i}(t)$. We suppose that all parameters and variables in Eq. (1.1) are dimensionless and that the noises are white with $\left\langle\xi_{i}(t)\right\rangle=0$ and

$$
\left\langle\xi_{i}(t) \xi_{j}\left(t^{\prime}\right)\right\rangle=2 \Delta_{i j} \delta\left(t-t^{\prime}\right) .
$$

Here $\langle\ldots\rangle$ denotes averaging with respect to the noises $\xi_{i}(t), \Delta_{11} \equiv \Delta_{1}(\geq 0)$ and $\Delta_{22} \equiv$ $\Delta_{2}(\geq 0)$ are the intensities of the noises $\xi_{1}(t)$ and $\xi_{2}(t)$, respectively, $\Delta_{12}=\Delta_{21} \equiv r \sqrt{\Delta_{1} \Delta_{2}}$, $r$ is the parameter characterizing the cross-correlation of the noises, $|r| \leq 1$, and $\delta(t)$ is the Dirac delta-function. For generality, we assume that the noises $\xi_{i}(t)$ are external and so the time-dependent friction and stochastic forces in Eq. (1.1) are not related to the fluctuation-dissipation theorem. Hence, if internal noises are negligible in comparison with external ones, this equation can be used, e.g., for the description of Brownian particles in a liquid with a time-dependent temperature. It is important to note, however, that Eq. (1.1) has a physical meaning for internal noises as well. Indeed, since according to the Stokes formula the friction coefficient depends on the particle radius, this equation describes in the overdamped regime the Brownian dynamics of spherical particles with time-dependent radii. Therefore, Eq. (1.1) with internal noises can be used, e.g., for the study of stochastic dynamics of nucleating and reacting particles, see in this context also related works and the given applications therein which considers instead a fluctuating friction [43, 44]. Equation (1.1) does not include inertial effects since in most cases they are important only for a very short initial time interval and can be safely neglected for larger time scales.

The paper is organized as follows. In Sec. II, using the Langevin approach, we derive the time-dependent probability distribution function $(\mathrm{PDF})$ of $x(t)$ and calculate its moments. In Sec. III, we find the long-time asymptotic behavior for moments and classify the diffusive regimes of the particles. We obtain some exact solutions in Sec. IV. In the same section we compare the exact and asymptotic results. We summarize our results in Sec. IV. Finally, we derive the PDF by the Fokker-Planck method in the Appendix. 


\section{PROBABILITY DISTRIBUTION FUNCTION AND ITS MOMENTS}

According to the results of Refs. 34, 35, 45], the two-noise Langevin equation (1.1) is statistically equivalent to a one-noise Langevin equation. Its form depends on how Eq. (1.1) is interpreted [40]. In the case of the Stratonovich interpretation [46, 47] we obtain

$$
\lambda(t) \dot{x}(t)=G(x(t)) \zeta(t) \quad\left[x(0)=x_{0}\right]
$$

where

$$
G(x)=\sqrt{\sum_{i=1}^{2} \sum_{j=1}^{2} \Delta_{i j} g_{i}(x) g_{j}(x)}
$$

and $\zeta(t)$ is Gaussian white noise with zero mean and correlation function

$$
\left\langle\zeta(t) \zeta\left(t^{\prime}\right)\right\rangle=2 \delta\left(t-t^{\prime}\right)
$$

In general, the function $G(x)$ will be nonnegative. Here, we consider the slightly more stringent case that $G(x)>0$ for all $x$, and consequently $x(t) \in(-\infty, \infty)$.

In order to find the PDF of $x(t)$, we use an explicit expression for $x(t)$ that follows from Eq. (2.1). (An alternative derivation of the PDF via the solution of the corresponding Fokker-Planck equation is presented in the Appendix.) The Wang and Zakai theorem [47, 48] states that the conventional rules of calculus are applicable to Langevin equations if they are interpreted as Stratonovich stochastic equations. Accordingly we solve for $x(t)$ by separating the variables and integrating Eq. (2.1) to obtain

$$
\int_{x_{0}}^{x(t)} \frac{d x^{\prime}}{G\left(x^{\prime}\right)}=\int_{0}^{t} \frac{\zeta\left(t^{\prime}\right) d t^{\prime}}{\lambda\left(t^{\prime}\right)} .
$$

If we define

$$
\Psi(x)-\Psi\left(x_{0}\right)=\int_{x_{0}}^{x} \frac{d x^{\prime}}{G\left(x^{\prime}\right)}
$$

and

$$
w(t)=\int_{0}^{t} \frac{\zeta\left(t^{\prime}\right) d t^{\prime}}{\lambda\left(t^{\prime}\right)},
$$

then Eq. (2.4) can be rewritten in the form

$$
\Psi(x(t))=w(t)+u_{0}
$$

$\left[u_{0}=\Psi\left(x_{0}\right)\right]$. According to the definition (2.5), the function $u=\Psi(x)$ is continuous and monotonically increasing; consequently, we obtain $\min u=\Psi(-\infty)$, $\max u=\Psi(\infty)$. On 
the other hand, the range of the function $w(t)$ is over all real-valued numbers. Therefore, Eq. (2.7) is applicable at all times only if $\Psi(-\infty)=-\infty$ and $\Psi(\infty)=\infty$. We assume that these conditions are met and take into account that the inverse function $x=\Psi^{-1}(u)$ is single-valued, i.e. it is also continuous and monotonically increasing, to obtain the unique solution of Eq. (2.1) in the form

$$
x(t)=\Psi^{-1}\left(w(t)+u_{0}\right) .
$$

The relation (2.8) shows that the statistical properties of $x(t)$ are defined by the Gaussian process $w(t)$, which is fully characterized by its zero mean and the correlation function

$$
\left\langle w(t) w\left(t^{\prime}\right)\right\rangle=2 \int_{0}^{\min \left(t, t^{\prime}\right)} \frac{d \tau}{\lambda^{2}(\tau)} .
$$

Let $P_{x}(x, t)$ and $P_{w}(w, t)$ be the univariate PDFs that $x(t)=x$ and $w(t)=w$, respectively. Since a one-to-one correspondence exists between $x(t)$ and $w(t)$, the condition $P_{x}(x, t)|d x|=$ $P_{w}(w, t)|d w|$ must hold. Taking into account that $P_{w}(w, t)=[\sqrt{2 \pi} \sigma(t)]^{-1} \exp \left[-w^{2} / 2 \sigma^{2}(t)\right]$, where

$$
\sigma^{2}(t)=2 \int_{0}^{t} \frac{d \tau}{\lambda^{2}(\tau)}
$$

is the dispersion of $w(t)$, and using the relation that $d x / d w=G(x)$, which follows from Eqs. (2.5) and (2.7), we obtain for the desired PDF:

$$
P_{x}(x, t)=\frac{1}{\sqrt{2 \pi} G(x) \sigma(t)} \exp \left[-\frac{\left(\Psi(x)-u_{0}\right)^{2}}{2 \sigma^{2}(t)}\right] .
$$

This distribution function is properly normalized, namely, $\int_{-\infty}^{\infty} P_{x}(x, t) d x=1$, and its moments, defined as

$$
\left\langle x^{n}(t)\right\rangle=\int_{-\infty}^{\infty} d x x^{n} P_{x}(x, t)
$$

$(n=1,2, \ldots)$, contain valuable information about the stochastic system dynamics. Substituting Eq. (2.11) into Eq. (2.12) and introducing the new variable $y=\left(\Psi(x)-u_{0}\right) / \sigma(t)$, we reduce Eq. (2.12) into the convenient form

$$
\left\langle x^{n}(t)\right\rangle=\frac{1}{\sqrt{2 \pi}} \int_{-\infty}^{\infty} d y\left[\Psi^{-1}\left(y \sigma(t)+u_{0}\right)\right]^{n} e^{-y^{2} / 2} .
$$

Depending on the time-dependence of the friction coefficient $\lambda(t)$, the particles can be localized or delocalized as $t \rightarrow \infty$. If $\sigma(\infty)<\infty$, i.e., if $\lambda(t)$ grows fast enough, then the stationary $\operatorname{PDF} P_{x}(x, \infty)$ exists, and the particles are localized. Brownian particles in a freezing liquid serve as an example. On the contrary, if $\sigma(t) \rightarrow \infty$ as $t \rightarrow \infty$, then the particles are delocalized. We focus on the latter situation in the following. 


\section{ASYMPTOTIC BEHAVIOR FOR THE MOMENTS}

To study the long-time behavior of $x(t)$, including various diffusive regimes, we derive the asymptotic behavior of $\left\langle x^{n}(t)\right\rangle$ for $t \rightarrow \infty$. This is a rather complex problem because the integrand in (2.13) contains the inverse function $\Psi^{-1}(u)$ of the integral function $\Psi(x)$. Since the dispersion $\sigma^{2}(t)$ tends to infinity in the long-time limit, the inverse function at $y>0$ and $y<0$ can be replaced by its asymptotics at $u \rightarrow \infty$ and $u \rightarrow-\infty$, respectively. To find these asymptotic behaviors, we assume that

$$
G(x) \sim \sqrt{\Delta_{\rho}}|x|^{\alpha_{\rho}} \quad(x \rightarrow \rho \infty)
$$

where $\rho=+$ or,$- \Delta_{\rho}$ is the effective white noise intensity at $x=\rho \infty$, and $\alpha_{\rho} \leq 1$ to ensure that $\Psi( \pm \infty)= \pm \infty$. Then for $x \rightarrow \rho \infty$, Eq. (2.5) yields

$$
\Psi(x) \sim \frac{\rho}{\sqrt{\Delta_{\rho}}}\left\{\begin{array}{l}
\ln \frac{|x|}{a_{\rho}}, \quad \alpha_{\rho}=1, \\
\frac{|x|^{1-\alpha_{\rho}}}{1-\alpha_{\rho}}, \quad \alpha_{\rho}<1 .
\end{array}\right.
$$

[The normalizing parameter $a_{\rho}$ can be found if the explicit form of the function $\Psi(x)$ is known (see Sec. IV).] Finally, from Eq. (3.2) we obtain

$$
\Psi^{-1}(u) \sim \rho a_{\rho} \exp \left(\sqrt{\Delta_{\rho}}|u|\right) \quad(u \rightarrow \rho \infty)
$$

for $\alpha_{\rho}=1$, and

$$
\Psi^{-1}(u) \sim \rho\left[\sqrt{\Delta_{\rho}}\left(1-\alpha_{\rho}\right)|u|\right]^{\frac{1}{1-\alpha_{\rho}}} \quad(u \rightarrow \rho \infty)
$$

for $\alpha_{\rho}<1$. Next we consider separately these two cases.

$$
\text { 1. } \alpha_{\rho}=1
$$

In this case, we replace the inverse function $\Psi^{-1}\left(y \sigma(t)+u_{0}\right)$ by its asymptotic behavior, i.e., $a_{+} \exp \left[\sqrt{\Delta_{+}}\left(y \sigma(t)+u_{0}\right)\right]$ at $y>0$ and $-a_{-} \exp \left[-\sqrt{\Delta_{-}}\left(y \sigma(t)+u_{0}\right)\right]$ at $y<0$, and Eq. (2.13) thus yields

$$
\begin{aligned}
\left\langle x^{n}(t)\right\rangle \sim & \frac{1}{\sqrt{2 \pi}} \sum_{\rho}\left(\rho a_{\rho}\right)^{n} \int_{0}^{\infty} d y \exp \left(n \sqrt{\Delta_{\rho}}(y \sigma(t)\right. \\
& \left.\left.+\rho u_{0}\right)-y^{2} / 2\right)
\end{aligned}
$$


as $t \rightarrow \infty$. Using the standard integral formula [49]

$$
\int_{0}^{\infty} d y e^{-p y-y^{2} / 2}=\sqrt{\frac{\pi}{2}} e^{p^{2} / 2} \operatorname{erfc}\left(\frac{p}{\sqrt{2}}\right)
$$

where $\operatorname{erfc}(z)=(2 / \sqrt{\pi}) \int_{z}^{\infty} d t e^{-t^{2}}$ is the complementary error function, and taking into account the relation $\operatorname{erfc}(-\infty)=2$, we reduce the asymptotic formula (3.5) to the form

$$
\left\langle x^{n}(t)\right\rangle \sim \sum_{\rho}\left(\rho a_{\rho}\right)^{n} \exp \left(\rho n \sqrt{\Delta_{\rho}} u_{0}+n^{2} \Delta_{\rho} \sigma^{2}(t) / 2\right) .
$$

It is important to note that, since (3.3) represents the leading term of the asymptotic expansion of $\Psi^{-1}(u)$, we should keep only the largest term in the right-hand side of (3.7). In particular, if $\Delta_{+}>\Delta_{-}$then

$$
\left\langle x^{n}(t)\right\rangle \sim a_{+}^{n} \exp \left(n \sqrt{\Delta_{+}} u_{0}+n^{2} \Delta_{+} \sigma^{2}(t) / 2\right)
$$

$(t \rightarrow \infty)$, i.e., the particles tend to plus infinity, and if $\Delta_{+}<\Delta_{-}$then

$$
\left\langle x^{n}(t)\right\rangle \sim(-1)^{n} a_{-}^{n} \exp \left(-n \sqrt{\Delta_{-}} u_{0}+n^{2} \Delta_{-} \sigma^{2}(t) / 2\right)
$$

$(t \rightarrow \infty)$, i.e., the particles tend to minus infinity. We emphasize that such a behavior of $x(t)$ results as a direct consequence of the multiplicative nature of the noise.

If $\Delta_{+}=\Delta_{-}=\Delta$ then $a_{+}=a_{-}=a$. Both terms in the right-hand side of (3.7) have the same order, and the asymptotic relation (3.7) is reduced to

$$
\left\langle x^{n}(t)\right\rangle \sim 2 a^{n} \cosh \left(n \sqrt{\Delta} u_{0}\right) \exp \left(n^{2} \Delta \sigma^{2}(t) / 2\right)
$$

$(t \rightarrow \infty)$ for even $n$ and to

$$
\left\langle x^{n}(t)\right\rangle \sim 2 a^{n} \sinh \left(n \sqrt{\Delta} u_{0}\right) \exp \left(n^{2} \Delta \sigma^{2}(t) / 2\right)
$$

$(t \rightarrow \infty)$ for odd $n$. The last relation shows that, in contrast to the previous discussed cases, no systematic growth of $\langle x(t)\rangle$ occurs for $u_{0}=0$, i.e., $x_{0}=\Psi^{-1}(0)$. In other words, in this case the particle position $x(t)$ exhibits purely diffusive behavior that can be characterized by the dispersion $\sigma_{x}^{2}(t)=\left\langle x^{2}(t)\right\rangle-\langle x(t)\rangle^{2}$. Since $\sigma_{x}^{2}(t) \sim 2 a^{2} e^{2 \Delta \sigma^{2}(t)}$ as $t \rightarrow \infty$, different long-time regimes of diffusion can exist.

We describe these regimes for the case that $\lambda(t) \sim l t^{\beta}(t \rightarrow \infty)$, where $l$ is a positive parameter and $\beta \leq 1 / 2$ (the last condition guarantees that $\sigma(\infty)=\infty$ ). Using Eq. (2.10), for $\beta<1 / 2$ we obtain

$$
\ln \sigma_{x}^{2}(t) \sim \frac{4 \Delta}{l^{2}(1-2 \beta)} t^{1-2 \beta}
$$


$(t \rightarrow \infty)$. Accordingly, we call the diffusive behavior a stretched exponential one if $0<$ $\beta<1 / 2$, exponential if $\beta=0$, and a compressed exponential if $\beta<0$. If $\beta=1 / 2$, then $\sigma^{2}(t) \sim\left(2 / l^{2}\right) \ln (t / \tilde{t})[\tilde{t}$ is some characteristic time scale] and so

$$
\sigma_{x}^{2}(t) \sim 2 a^{2}(t / \tilde{t})^{\frac{4 \Delta}{l^{2}}}
$$

as $t \rightarrow \infty$. This asymptotic formula demonstrates a truly remarkable feature of this physical system, namely: the character of its diffusive regime depends on the effective white noise intensity $\Delta$ which in the general case depends on the correlation coefficient $r$. Following the conventional terminology, we say that the system exhibits subdiffusion if $\Delta<l^{2} / 4$, normal diffusion if $\Delta=l^{2} / 4$, and superdiffusion if $\Delta>l^{2} / 4$. We expect that these diffusion regimes can exist in the systems of nucleating particles whose radii grow as $t^{1 / 2}$.

2. $\alpha_{\rho}<1$

In this case, using the standard integral formula [49]

$$
\int_{0}^{\infty} d y y^{\mu} e^{-y^{2} / 2}=2^{\frac{\mu-1}{2}} \Gamma\left(\frac{\mu+1}{2}\right)
$$

$\left[\mu>-1, \Gamma(z)=\int_{0}^{\infty} d x x^{z-1} e^{-x}\right.$ is the gamma function] and the asymptotic relation (3.4), Eq. (2.13) yields

$$
\begin{aligned}
\left\langle x^{n}(t)\right\rangle \sim & \frac{1}{2 \sqrt{\pi}} \sum_{\rho}(\rho 1)^{n} \Gamma\left(\eta_{\rho} / 2\right) \\
& \times\left[\sqrt{2 \Delta_{\rho}}\left(1-\alpha_{\rho}\right) \sigma(t)\right]^{\eta_{\rho}-1}
\end{aligned}
$$

$(t \rightarrow \infty)$, where $\eta_{\rho}=1+n /\left(1-\alpha_{\rho}\right)$. We emphasize that in this asymptotic relation only the dominant term on the right side should be kept. Specifically, if $\alpha_{+}>\alpha_{-}$then

$$
\left\langle x^{n}(t)\right\rangle \sim \frac{\Gamma\left(\eta_{+} / 2\right)}{2 \sqrt{\pi}}\left[\sqrt{2 \Delta_{+}}\left(1-\alpha_{+}\right) \sigma(t)\right]^{\eta_{+}-1},
$$

and if $\alpha_{+}<\alpha_{-}$then

$$
\left\langle x^{n}(t)\right\rangle \sim(-1)^{n} \frac{\Gamma\left(\eta_{-} / 2\right)}{2 \sqrt{\pi}}\left[\sqrt{2 \Delta_{-}}\left(1-\alpha_{-}\right) \sigma(t)\right]^{\eta_{-}-1} .
$$

Finally, if $\alpha_{\rho}=\alpha\left(\eta_{\rho}=\eta\right)$ then both terms have the same order and so

$$
\begin{aligned}
\left\langle x^{n}(t)\right\rangle \sim & \frac{\Gamma(\eta / 2)}{2 \sqrt{\pi}}\left[\Delta_{+}^{\frac{\eta-1}{2}}+(-1)^{n} \Delta_{-}^{\frac{\eta-1}{2}}\right] \\
& \times[\sqrt{2}(1-\alpha) \sigma(t)]^{\eta-1} .
\end{aligned}
$$


These relations show that for $\Delta_{+} \neq \Delta_{-}$all moments of $x(t)$ diverge as $t \rightarrow \infty$.

Next, we assume that $\alpha_{\rho}=\alpha$ and $\Delta_{\rho}=\Delta$. In this case, using the asymptotic relation (3.4), Eq. (2.13) for $t \rightarrow \infty$ is reduced to

$$
\left\langle x^{n}(t)\right\rangle \sim \frac{\Gamma(\eta / 2)}{\sqrt{\pi}}[\sqrt{2 \Delta}(1-\alpha) \sigma(t)]^{\eta-1}
$$

if $n$ is even, and to

$$
\left\langle x^{n}(t)\right\rangle \sim u_{0} \frac{\eta-1}{\sqrt{2 \pi}} \Gamma\left(\frac{\eta-1}{2}\right)[\sqrt{2 \Delta}(1-\alpha)]^{\eta-1} \sigma^{\eta-2}(t)
$$

if $n$ is odd. The last relation and the exact formula (2.13) confirm the fact that $\langle x(t)\rangle=0$ for $u_{0}=0$, i.e., under these conditions the particles display diffusive behavior. Since in this case

$$
\sigma_{x}^{2}(t) \sim \frac{\Gamma(1 /(1-\alpha)-1 / 2)}{\sqrt{\pi}}[\sqrt{2 \Delta}(1-\alpha) \sigma(t)]^{\frac{2}{1-\alpha}}
$$

as $t \rightarrow \infty$, its character depends on the exponent $\alpha$ and on the asymptotic behavior of $\sigma(t)$. Specifically, if $\lambda(t) \propto t^{\beta}(t \rightarrow \infty, \beta \leq 1 / 2)$ and so $\sigma^{2}(t) \propto t^{1-2 \beta}$ for $\beta<1 / 2$ and $\sigma^{2}(t) \propto \ln (t / \tilde{t})$ for $\beta=1 / 2$, then the particles exhibit superdiffusion if $\alpha>2 \beta$ and $\beta<1 / 2$, normal diffusion if $\alpha=2 \beta$ and $\beta<1 / 2$, subdiffusion if $\alpha<2 \beta$ and $\beta<1 / 2$, and logarithmic diffusion $\left[\sigma_{x}^{2}(t) \propto \ln ^{\frac{2}{1-\alpha}}(t / \tilde{t})\right]$ if $\beta=1 / 2$.

Thus, multiplicativity of noises and time dependency of the friction coefficient can give rise to anomalous behavior of the system. Specifically, if $\lambda=$ const then the conditions $\left.G(x)\right|_{x \rightarrow \pm \infty} \rightarrow \infty$ and $\left.G(x)\right|_{x \rightarrow \pm \infty} \rightarrow 0$ are responsible for the fast and slow diffusion, respectively. On the contrary, if $G(x)=$ const (white noises are additive) then diffusion is fast if $\left.\lambda(t)\right|_{t \rightarrow \infty} \rightarrow 0$ and is slow if $\left.\lambda(t)\right|_{t \rightarrow \infty} \rightarrow \infty$. Remarkable, the presence of both these factors can lead to new stochastic phenomena, like the dependence of the character of anomalous diffusion on the effective white noise intensity. We note also that the nature of anomalous diffusion in this system, due the features of the stochastic and friction forces mentioned above, is quite different from that observed for random walks with long-tail jump-length and/or waiting-time distributions.

\section{SPECIFIC EXAMPLES}

To confirm our asymptotic results, we calculate explicit the expressions for the moments of $x(t)$ for two particular cases. 


\section{A. Two additive noises}

As a first example, we consider the case that both white noises are additive, i.e., $g_{1}(x)=$ $g_{2}(x)=1$. Then, $G^{2}(x)=\Delta_{1}+\Delta_{2}+2 r \sqrt{\Delta_{1} \Delta_{2}} \equiv G^{2}, \Psi(x)=x / G, \Psi^{-1}(u)=G u$, $u_{0}=x_{0} / G$, and Eq. (2.13) is reduced to

$$
\left\langle x^{n}(t)\right\rangle=\frac{1}{\sqrt{2 \pi}} \int_{-\infty}^{\infty} d y\left[y G \sigma(t)+x_{0}\right]^{n} e^{-y^{2} / 2} .
$$

Using in (4.1) the binomial formula and taking into account the relation (3.14), we obtain after some calculations

$$
\left\langle x^{n}(t)\right\rangle=\frac{x_{0}^{n}}{\sqrt{\pi}} \sum_{k=0}^{[n / 2]} C_{n}^{2 k} \Gamma\left(k+\frac{1}{2}\right)\left[\frac{\sqrt{2} G}{x_{0}} \sigma(t)\right]^{2 k},
$$

where $[n / 2]$ is the integer part of $n / 2$ and $C_{n}^{m}=n ! /(n-m) ! m !$ is the binomial coefficient.

According to this formula, the leading term of the long-time asymptotic expansion of $\left\langle x^{n}(t)\right\rangle$ is given by the term in the sum that corresponds to $k=[n / 2]$, i.e.,

$$
\left\langle x^{n}(t)\right\rangle \sim \frac{1}{\sqrt{\pi}} \Gamma\left(\frac{n+1}{2}\right)[\sqrt{2} G \sigma(t)]^{n}
$$

for even $n$ and

$$
\left\langle x^{n}(t)\right\rangle \sim \frac{x_{0}}{\sqrt{\pi}} n \Gamma\left(\frac{n}{2}\right)[\sqrt{2} G \sigma(t)]^{n-1}
$$

for odd $n$. Since $\Delta=G^{2}, \alpha=0$ and $\eta=n+1$, the same asymptotic behavior follows also from the formulas (3.19) and (3.20), respectively.

\section{B. Additive and multiplicative noises}

As a second example, we consider a linear Langevin equation where one noise is multiplicative, $g_{1}(x)=x$, and the other one is additive, i.e., $g_{2}(x)=1$. According to the definition (2.2), in this case

$$
G(x)=\sqrt{\Delta_{1}\left(x^{2}+2 r \nu x+\nu^{2}\right)}
$$

$\left(\nu=\sqrt{\Delta_{2} / \Delta_{1}}\right)$ and for $|r|<1$ we obtain

$$
\Psi(x)=\frac{1}{\sqrt{\Delta_{1}}} \operatorname{arcsinh}\left(\frac{x+r \nu}{\nu \sqrt{1-r^{2}}}\right)
$$

and

$$
\Psi^{-1}(u)=\nu \sqrt{1-r^{2}} \sinh \left(\sqrt{\Delta_{1}} u\right)-r \nu
$$


Then, using Eqs. (2.13) and (4.7), the relation

$$
\sinh \left(\sqrt{\Delta_{1}} u_{0}\right)=\frac{x_{0}+r \nu}{\nu \sqrt{1-r^{2}}}
$$

that follows from Eq. (4.7), and the standard integral formula [49]

$$
\begin{aligned}
\int_{-\infty}^{\infty} d y e^{-b y^{2}+c y}\left[\begin{array}{c}
\sinh (p y) \\
\cosh (p y)
\end{array}\right]= & \sqrt{\frac{\pi}{b}} \exp \left(\frac{c^{2}+p^{2}}{4 b}\right) \\
& \times\left[\begin{array}{c}
\sinh (c p / 2 b) \\
\cosh (c p / 2 b)
\end{array}\right]
\end{aligned}
$$

$(b>0)$, we find the first moment

$$
\langle x(t)\rangle=\left(x_{0}+r \nu\right) \exp \left(\Delta_{1} \sigma^{2}(t) / 2\right)-r \nu,
$$

which shows that $x(t)$ exhibits purely diffusive behavior only if $r=-x_{0} / \nu$, and the second one

$$
\begin{aligned}
\left\langle x^{2}(t)\right\rangle= & {\left[\left(x_{0}+r \nu\right)^{2}+\nu^{2}\left(1-r^{2}\right) / 2\right] \exp \left[2 \Delta_{1} \sigma^{2}(t)\right] } \\
& -2 r \nu\left(x_{0}+r \nu\right) \exp \left(\Delta_{1} \sigma^{2}(t) / 2\right) \\
& +\nu^{2}\left(3 r^{2}-1\right) / 2
\end{aligned}
$$

For $t \rightarrow \infty$, these exact results readily yield

$$
\langle x(t)\rangle \sim\left(x_{0}+r \nu\right) \exp \left(\Delta_{1} \sigma^{2}(t) / 2\right)
$$

and

$$
\left\langle x^{2}(t)\right\rangle \sim\left[\left(x_{0}+r \nu\right)^{2}+\nu^{2}\left(1-r^{2}\right) / 2\right] \exp \left[2 \Delta_{1} \sigma^{2}(t)\right] .
$$

On the other hand, the same long-time representations for $\langle x(t)\rangle$ and $\left\langle x^{2}(t)\right\rangle$ follow as well from the more general asymptotic formulas (3.10) and (3.11). To verify this, we compare (3.1) with the asymptotic relation $G(x) \sim \sqrt{\Delta_{1}}|x|$, which follows from Eq. (4.5) as $|x| \rightarrow \infty$; this yields $\Delta_{\rho}=\Delta=\Delta_{1}$ and $\alpha_{\rho}=\alpha=1$. Next, since $\operatorname{arcsinh} z \sim \rho \ln 2|z|$ as $z \rightarrow \rho \infty$, from Eq. (4.6) we find the asymptotic formula

$$
\Psi(x) \sim \frac{\rho}{\sqrt{\Delta_{1}}} \ln \left(\frac{2|x|}{\nu \sqrt{1-r^{2}}}\right)
$$


$(x \rightarrow \rho \infty)$, which by comparison with (3.2) yields $a_{\rho}=a=\nu \sqrt{1-r^{2}} / 2$. Finally, substituting the values for $\Delta$ and $a$ into the formulas (3.11) [with $n=1]$ and (3.10) [with $n=2$ ] and using the relation (4.8), we indeed recover the asymptotic behavior (4.12) and (4.13).

Thus, our exact results corroborate the general asymptotic formulas obtained in the previous section.

\section{CONCLUSIONS}

We have studied analytically the statistical properties of a special class of exactly solvable stochastic models represented by an overdamped particle that is driven by two crosscorrelated multiplicative Gaussian white noises in a time-dependent environment. As a first step, we have reduced the initial two-noise Langevin equation that describes the particle dynamics in the Stratonovich sense to a stochastically equivalent one-noise Langevin equation. Then, solving the latter equation and the corresponding Fokker-Planck equation, we have derived the exact probability distribution function for the particle positions.

To study the long-time behavior of the particle dynamics, we have calculated the asymptotic behavior for the moments of the distribution function. Their analysis results in a rich behavior of differing regimes of anomalous particle diffusion; namely we find regimes with normal diffusion, subdiffusion, superdiffusion, exponential diffusion, stretched exponential diffusion, compressed exponential diffusion, and, as well, logarithmic diffusion. We have established conditions for these diffusion behaviors to occur and in some special cases we were able to derive exact formulas for the moments of order 2 and higher that confirmed the general asymptotic results. Also, we have discovered the truly remarkable feature for systems described by a special class of linear Langevin equations that their diffusive behavior becomes determined by the effective white noise intensity.

\section{ACKNOWLEDGMENTS}

S.I.D. acknowledges the support of the European Union through a Marie Curie individual fellowship, contract MIF1-CT-2005-007021, and P.H. the support of the Deutsche Forschungsgemeinschaft, grant HA 1517/13-4. 


\section{APPENDIX: ALTERNATIVE DERIVATION OF THE PDF (2.11)}

To derive the PDF (2.11) by the Fokker-Planck method, we assume that each Gaussian white noise $\xi_{i}(t)$ is characterized by its own parameter $\gamma_{i}\left(0 \leq \gamma_{i} \leq 1\right)$ that determines the points of time at which $g_{i}(x(t))$ is evaluated in the corresponding integral sum. Then the Fokker-Planck equation associated with the Langevin equation (1.1) takes the form [40]

$$
\lambda^{2}(t) \frac{\partial}{\partial t} P_{x}(x, t)=-\frac{\partial}{\partial x} h(x) P_{x}(x, t)+\frac{\partial^{2}}{\partial x^{2}} d(x) P_{x}(x, t)
$$

$\left[P_{x}(x, 0)=\delta\left(x-x_{0}\right)\right]$, where

$$
h(x)=2 \sum_{i=1}^{2} \sum_{j=1}^{2} \gamma_{i} \Delta_{i j} g_{i}^{\prime}(x) g_{j}(x)
$$

is the drift coefficient,

$$
d(x)=\sum_{i=1}^{2} \sum_{j=1}^{2} \Delta_{i j} g_{i}(x) g_{j}(x),
$$

is the diffusion coefficient, and the prime denotes the derivative with respect to the argument of the function.

In order to solve Eq. A.1 , we introduce a new variable $u=U(x)$, where the function $U(x)$ remains to be defined. If the functions $U(x)$ and $U^{-1}(u)$ are single-valued and $U^{\prime}(x)>0$, then

$$
P_{x}(x, t)=P_{u}(u, t) U^{\prime}(x) .
$$

According to Eq. (A.1), the PDF $P_{u}(u, t)$ of the random process $u(t)=U(x(t))$ satisfies the Fokker-Planck equation whose drift and diffusion coefficients (as functions of the old variable $x$ ) are given by

$$
\tilde{h}(x)=h(x) U^{\prime}(x)+d(x) U^{\prime \prime}(x), \quad \tilde{d}(x)=d(x)\left[U^{\prime}(x)\right]^{2} .
$$

If we chose $\tilde{d}(x)=1$, then $U^{\prime}(x)=1 / \sqrt{d(x)}$ and

$$
\tilde{h}(x)=\frac{2 h(x)-d^{\prime}(x)}{2 \sqrt{d(x)}} .
$$

Remarkably, if $\gamma_{i}=1 / 2$, i.e., if the Stratonovich interpretation of the Langevin equation (1.1) is used, then $\tilde{h}(x)=0$ and the Fokker-Planck equation for $P_{u}(u, t)$ takes the simplest form

$$
\lambda^{2}(t) \frac{\partial}{\partial t} P_{u}(u, t)=\frac{\partial^{2}}{\partial u^{2}} P_{u}(u, t)
$$


Solving it, for example, by the generating function method and using the initial condition $P(u, 0)=\delta\left(x-x_{0}\right) \sqrt{d(x)}\left[\right.$ recall that $\left.x=U^{-1}(u)\right]$, we find

$$
P_{u}(u, t)=\frac{1}{\sqrt{2 \pi} \sigma(t)} \exp \left[-\frac{\left(u-u_{0}\right)^{2}}{2 \sigma^{2}(t)}\right] .
$$

Finally, substituting this result into Eq. (A.4) and taking into account that $U(x)=\Psi(x)$, $d(x)=G^{2}(x)$ and $u=\Psi(x)$, we again obtain Eq. (2.11).

[1] N. G. Van Kampen, Stochastic Processes in Physics and Chemistry (North-Holland, Amsterdam, 1992).

[2] W. Horsthemke and R. Lefever, Noise-Induced Transitions (Springer-Verlag, Berlin, 1984).

[3] C. W. Gardiner, Handbook of Stochastic Methods, 2nd ed. (Springer-Verlag, Berlin, 1990).

[4] J. P. Bouchaud and A. Georges, Phys. Rep. 195, 127 (1990).

[5] D. ben-Avraham and S. Havlin, Diffusion and Reactions in Fractals and Disordered Systems (Cambridge University Press, Cambridge, 2000).

[6] G. Zaslavsky, Phys. Rep. 371, 461 (2002).

[7] R. Metzler and J. Klafter, J. Phys. A: Math. Gen. 37 R161 (2004).

[8] W. R. Schneider and W. Wyss, J. Math. Phys. 30, 134 (1989).

[9] R. Hilfer, Fractals 3, 211 (1995); R. Hilfer, Fractional calculus in physics, (World Scientific, Singapore, 2000).

[10] R. Metzler and J. Klafter, Phys. Rep. 339, 1 (2000).

[11] H. C. Fogedby, Phys. Rev. E 50, 1657 (1994).

[12] A. Compte, Phys. Rev. E 53, 4191 (1996).

[13] R. Metzler, E. Barkai, and J. Klafter, Phys. Rev. Lett. 82, 3563 (1999).

[14] E. Barkai, Phys. Rev. E 63, 046118 (2001).

[15] V. Kobelev and E. Romanov, Prog. Theor. Phys. Suppl. 139, 470 (2000).

[16] E. Lutz, Phys. Rev. E 64, 051106 (2001).

[17] A. Bazzani, G. Bassi, and G. Turchetti, Physica A 324, 530 (2003).

[18] P. Hänggi, Phys. Lett. A 83, 196 (1981).

[19] B. O’Shaughnessy and I. Procaccia, Phys. Rev. Lett. 54, 455 (1985).

[20] R. Muralidhar, D. Ramkrishna, H. Nakanishi, and D. Jacobs, Physica A 167, 539 (1990). 
[21] J. M. Porrà, K. G. Wang, and J. Masoliver, Phys. Rev. E 53, 5872 (1996).

[22] K. G. Wang and M. Tokuyama, Physica A 265, 341 (1999).

[23] R. Morgado, F. A. Oliveira, G. G. Batrouni, and A. Hansen, Phys. Rev. Lett. 89100601 (2002).

[24] J. D. Bao and Y. Z. Zhuo, Phys. Rev. E 71, 010102(R) (2005).

[25] J. D. Bao, P. Hänggi, and Y. Z. Zhuo, Phys. Rev. E 72, 061107 (2005).

[26] A. V. Mokshin, R. M. Yulmetyev, and P. Hänggi, Phys. Rev. Lett. 95, 200601 (2005).

[27] J. Masoliver, Phys. Rev. A 45, 706 (1992).

[28] J. Heinrichs, Phys. Rev. E 47, 3007 (1993).

[29] J. Masoliver and K. G. Wang, Phys. Rev. E 51, 2987 (1995).

[30] S. I. Denisov and W. Horsthemke, Phys. Rev. E 62, 7729 (2000).

[31] S. I. Denisov and W. Horsthemke, Phys. Rev. E 65, 031105 (2002).

[32] A. N. Vitrenko, Physica A 359, 65 (2006).

[33] F. Lillo and R. N. Mantegna, Phys. Rev. E 61, R4675 (2000).

[34] A. Fulinski and T. Telejko, Phys. Lett. A 152, 11 (1991).

[35] A. J. R. Madureira, P. Hänggi, and H. S. Wio, Phys. Lett. A 217, 248 (1996).

[36] L. Gammaitoni, P. Hänggi, P. Jung, and F. Marchesoni, Rev. Mod. Phys. 70, 223 (1998).

[37] P. Hänggi, ChemPhysChem 3, 285 (2002).

[38] J. H. Li and P. Hänggi, Phys. Rev. E 64, 011106 (2001).

[39] Y. Jia and J. R. Li, Phys. Rev. Lett. 78, 994 (1997).

[40] S. I. Denisov, A. N. Vitrenko, and W. Horsthemke, Phys. Rev. E 68, 046132 (2003).

[41] L. Cao and D. J. Wu, Phys. Rev. E 62, 7478 (2000).

[42] J. H. Li, J. Łuczka, and P. Hänggi, Phys. Rev. E 64, 011113 (2001).

[43] J. Łuczka, P. Talkner, and P. Hänggi, Physica A 278, 18 (1999).

[44] J. Łuczka, P. Hänggi, and A. Gadomski, Phys. Rev. E 51, 5762 (1995).

[45] D. J. Wu, L. Cao, and S. Z. Ke, Phys. Rev. E 50, 2496 (1994).

[46] R. L. Stratonovich, SIAM J. Control 4, 362 (1966).

[47] P. Hänggi and H. Thomas, Phys. Rep. 88, 207 (1982); see Sec. 2.4.

[48] E. Wong and M. Zakai, Ann. Math. Stat. 36, 1560 (1965).

[49] A. P. Prudnikov, Yu. A. Brychkov, and O. I. Marichev, Integrals and Series (Gordon \& Breach, New York, 1989-1990), Vols. 1-3. 